\title{
New diabetes nutrition recommendations-food for thought
}

\section{T} wo new publications provide guidance on healthy eating for patients with diabetes mellitus and their health-care providers. The American Diabetes Association (ADA) have updated their previous position statement, largely on the basis of a systematic review published in 2012 and literature published thereafter, and focus on nutrition therapy recommendations for the management of adults with type 1 and type 2 diabetes mellitus. Prevention of diabetes mellitus was not addressed in this position statement. Guidelines from the American Association of Clinical Endocrinologists (AACE) and The Obesity Society (TOS) target a broader audience, by providing evidence-based clinical practice guidelines to define standards of care for healthy eating both in the management and in the prevention of various metabolic and endocrine disorders, including but not restricted to diabetes mellitus.

Medical nutrition therapy is a pivotal component of health care in general and more specifically for diabetes management. However, a variety of obstacles preclude implementation of healthy eating strategies, including sociocultural factors.

Both publications recommend that nutrition therapy be provided-by a physician, physician extender, registered dietician and/or a certified diabetes educator-to all individuals with diabetes mellitus as an effective component of a diabetes treatment plan. Moreover, the ADA recommendations strongly emphasize the need for an individualized meal plan to achieve nutrition therapy goals, such as attaining individualized glycaemic, blood pressure and lipid targets, losing or maintaining body weight and delaying or preventing diabetes complications. "A variety of eating patterns (combinations of different foods or food groups) are acceptable for the management of diabetes mellitus, for example,

Mediterranean, low-fat, low-carbohydrate or vegetarian eating patterns," says Jackie L. Boucher (Minneapolis Heart

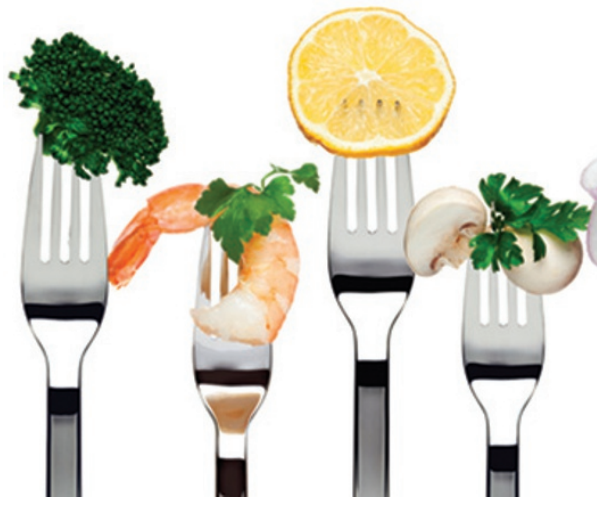

Institute Foundation), who is one of the corresponding authors of the ADA position statement. "The ADA recognizes that personal preferences and metabolic goals need to be considered and an eating plan developed collaboratively between the health-care professional and the individual with diabetes mellitus."

The ADA finds that evidence regarding the ideal amount of carbohydrate, protein and fat intake for individuals with diabetes mellitus is inconclusive. Here, the position statement differs from the AACE and TOS guidelines. Their guidelines recommend that carbohydrates account for about $45-65 \%$, protein for about $15-35 \%$ and total fat intake for about $30 \%$ of total daily calories in patients with diabetes mellitus. However, AACE and TOS do acknowledge that the clinical evidence for the carbohydrates and protein intake recommendations is weak; for fat intake, the evidence was graded 'intermediate'.

Evidence does not support recommending long-chain omega-3 fatty acid (EPA and DHA) supplements for people with diabetes for the prevention or treatment of cardiovascular events, according to the ADA position paper. However, in agreement with the AACE and TOS guidelines and as recommended for the general public, patients with diabetes mellitus should increase their consumption of foods containing omega- 3 fatty acids, for example, fatty fish.

Both task forces are in agreement that there is insufficient evidence to support

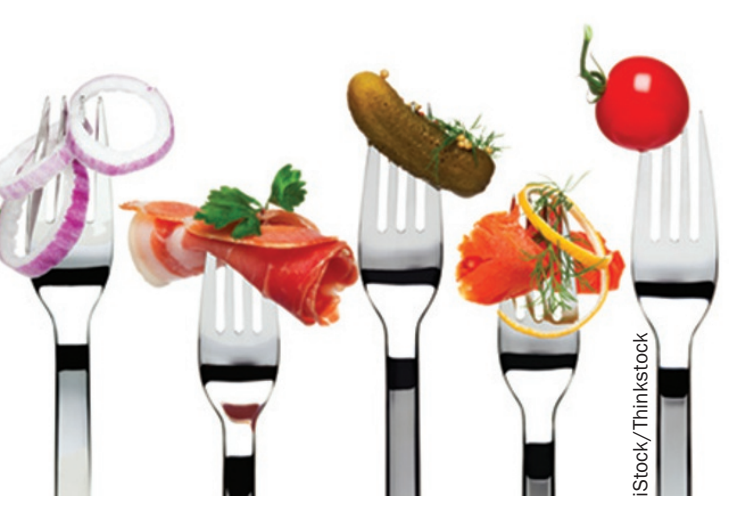

the routine use of antioxidants, such as vitamins $\mathrm{E}$ and $\mathrm{C}$ and carotene, or micronutrients, such as chromium and magnesium, in patients with diabetes mellitus. Moreover, alcohol consumption in patients with diabetes mellitus should be limited to a maximum of one or two drinks per day for women and men, respectively.

"More evidence on nutrition (individual nutrients and eating patterns) is needed to make more definitive recommendations for people with diabetes mellitus on what and when to eat," says Boucher. "People with diabetes mellitus should be able to eat the same healthful foods as everyone else to achieve nutrition therapy goals."

Taken together, the recommendations highlight the importance of collaborating with patients with diabetes mellitus to educate these individuals on healthy eating and proper meal planning. "Individuals with diabetes mellitus should receive an individualized eating plan that integrates lifestyle, culture and beliefs," concludes Boucher.

\section{Linda Koch}

